\title{
Remote Sensing of the Geomagnetic Tail Current Sheet Topology Using Energetic Ions: Neutral Lines versus Weak Field Regions
}

\author{
T. W. SPEISER ${ }^{1}$ and R. F. MARTIN, Jr. ${ }^{2}$ \\ ${ }^{1}$ Space Science Institute, Astrophysical, Planetary and Atmospheric Sciences Department/University of Colorado, and \\ Space Environment Laboratory/NOAA, Boulder, Colorado 80303-7814, U.S.A. \\ ${ }^{2}$ Physics Department, Campus Box 4560, Illinois State University, Normal, Illinois, 61790-4560, U.S.A.
}

(Received September 14, 1995; Revised February 5, 1996; Accepted March 19, 1996)

\begin{abstract}
Individual flow bursts in a bursty bulk flow event observed by the AMPTE/IRM satellite at $\boldsymbol{R}=$ $(-12,-3,1) R_{e}$, have been modeled by following large numbers of single particle orbits in a model of the geomagnetic tail current sheet containing both $B_{z}$ and $B_{y}$ components and a near Earth neutral line. A flow burst modeled in the central plasma sheet, just after a substorm onset, implied there was a near-Earth neutral line 1 to $1 \frac{1}{2} R_{e}$ tailward of the satellite. An earlier flow burst at the plasma sheet edge, 1-2 minutes before the substorm onset, implied that at this earlier time the neutral line was already formed and closer to the satellite. To match the centroid of the observations, it was necessary that the source population was strongly earthward and duskward flowing, probably originating from the distant current sheet, and that there must have been a relatively large $\left|B_{y}\right|$ component. With such a $B_{y}$ component, it is interesting that a secondary feature of the observed distribution can also be explained qualitatively. During the time that we see the need for a strong earthward flow from a distant source, ground measurements indicate a significant increase in magnetospheric convection. A model with weak but non-reversing $B_{z}$ reproduces some of the observed distribution function features, but not all of them, as well as the neutral line model. However, a secondary ridge seen in the observations implies a mixing of neutral line and weak field topologies, with such mixing occurring on a 1 minute time scale.
\end{abstract}

\section{Introduction}

In earlier studies (Martin and Speiser, 1988, 1994; Speiser and Martin, 1992, 1995; Martin et al., 1994; Dusenbery et al., 1996; Speiser et al., 1996), we have demonstrated the technique of following ion orbits backwards in time in model fields representing a quasi-steady current sheet (CS) in the geomagnetic tail. The model contains a neutral line at some distance from the assumed ending location of the particle orbits. In this model, a 2D neutral line is attached to a constant $B_{z}$ current sheet with $B_{z}$ at the center northward on the earthward $(x>0)$ side of the neutral line, and southward on the tailward $(x<0)$ side. The current sheet thickness is $2 T$ and the neutral line width in the $x$-direction is $2 L . T$ and $L$ are related by $T / L=$ $B_{z 0} / B_{x 0}$, where $B_{z 0}$ is the magnitude of the normal component and $B_{x 0}$ is the magnitude of the $B_{x}$ component for $|x| \geq L,|z|>T$. For our simulations, we specify $T, B_{x 0}, B_{z 0}$, and $E_{y}$, the assumed cross-tail electric field. Note that for $E_{y}>0$ (our general assumption), the model mimics reconnection, i.e., $E \cdot j>0$ throughout the CS. Also note that $E_{y}$ cannot be transformed away, as $B_{z}$ is not constant, i.e. there is no unique DeHoffmanTeller frame, in contrast to some earlier studies. For each simulation run, the Lorentz force equations are numerically integrated backwards in time, with orbits started at an observation point and followed until they leave the CS. On exit, orbits can be tagged with a source distribution, and the source distribution then mapped back to the observation point, using the Liouville Theorem. To tag each ion orbit with a value of an assumed initial distribution function we typically assume earthward or tailward flowing Maxwellian or kappa (Martin and Speiser, 1988) distributions. Using the Liouville theorem, we then map this source distribution to the ending location, the observation point of a satellite. After launching many particle orbits 
on a velocity grid at the ending location, we can then build up a map of the modeled distribution function to compare with the satellite's observed distribution function. For these simulations we use a grid of 25 $\times 25 \times 25$ on $V_{x}, V_{y}, V_{z}$, i.e., 15,625 particle orbits for each run. By varying parameters in the model and in the assumed source distribution, we try to model key features and asymmetries in the observed distribution function. We have previously modeled two distribution functions observed by the AMPTE/ IRM satellite in the geomagnetic tail near the onset time of a moderate substorm (Speiser and Martin, 1995; Speiser et al., 1996).

Baumjohann et al. (1990), reported that although the average flow speed in the central plasma sheet (CPS) is low, there are occasions when flows greater than $400 \mathrm{~km} / \mathrm{s}$ are observed. There is only a slight correlation with magnetic activity, with high speed flows being found about $1 \%$ of the time for low $A E$ ( $<300 \mathrm{nT}$ ), and $6 \%$ of the time for high $A E(>600 \mathrm{nT})$. An earlier high speed flow event was reported by Huang et al. (1987), near the neutral sheet during active periods. "Bursty bulk flows" are enhanced bulk velocity events of order 10 minutes in duration, and which contain typically many short-lived $(<10 \mathrm{sec})$, high velocity flow bursts, with flows greater than $400 \mathrm{~km} / \mathrm{s}$ (Angelopoulos et al., 1992). The flows usually occur near midnight in the CPS, mostly in the inner CPS, but sometimes in the outer CPS. The flows are predominantly earthward, and are often associated with local magnetic field variability and transient dipolarizarion. One such event has been published by Nakamura et al. (1991), for 1 March 1985, as observed by the AMPTE/IRM satellite. For this event, Nakamura et al. published a 3D distribution function in three Cartesian velocity planes, for the time 02:45:19. A moderate substorm occurred at 02:42, as evidenced by a jump in the $A E$ index from 100 to $400 \mathrm{nT}$. Rostoker (private communication, 1994), noted from inspection of the Great Whale River (GWR) magnetogram that magnetospheric convection picked up significantly at 02:30 UT and that there was an expansive phase onset at or very close to 02:42 UT, "the next notable happening after the 02:30 UT increase in convection". Friis-Christensen (private communication, 1994), also found that for the Greenland magnetometer chain $\left(76^{\circ}\right.$ to $86^{\circ}$, invariant latitudes), nearly at the same local time as AMPTE, that the earliest indication of the onset was at NAQ at about $02: 42 \mathrm{UT}(\Delta H$ at NAQ eventually reached about $-600 \mathrm{nT}$ at $0400 \mathrm{UT})$. At GOES $5\left(6.6 R_{e}, 75.8^{\circ} \mathrm{W}\right.$ longitude, about 10 PM LT), there was an indication of an onset of Pi 2-like activity between 02:42 UT and 02:43 UT. This substorm was followed by a storm interval lasting for 9 hours (Singer, private communication, 1994). For the 02:45:19 distribution, the flow speed was $522 \mathrm{~km} / \mathrm{s}$, earthward and dawnward (about half the thermal speed $\sim 1000 \mathrm{~km} / \mathrm{s}$ ) and the magnetic field was $1.6 \mathrm{nT}$, northward. The flow was thus subsonic but much higher than a typical Alfvén speed $(\sim 150 \mathrm{~km} / \mathrm{s})$. After the neutral sheet encounter the magnetic field becomes more dipolar. The first AMPTE distribution investigated was at 3 minutes after substorm onset, when AMPTE passed through the CS center at 02:45:19UT. In our modeling we found we could fairly well model the shape and bulk flow of the observed distribution $(522 \mathrm{~km} / \mathrm{s}$ earthward and dawnward). In order to match the shape and bulk flow, we needed a source kappa distribution that was flowing strongly earthward $(1,200 \mathrm{~km} / \mathrm{s})$ and duskward $(800 \mathrm{~km} / \mathrm{s})$. In order to match the ridge-like feature of the observed distribution function, the model neutral line had to be about 10,000 $\mathrm{km}$ tailward of the AMPTE observation point, $\boldsymbol{R}=(-12,-3,1) R_{e}$, in the CS center (Speiser and Martin, 1995). The model was therefore telling us that 3 minutes after a moderate substorm onset, there was a nearEarth neutral line at $X \simeq-13$ to $-14 R_{e}$, and that plasma from a distant tail source, maybe a distant neutral line, or the distant CS, was strongly accelerated in the earthward direction. (We do not know how distant the source is; it could be just a few $R_{e}$ tailward.)

The other distribution previously studied was one at 02:40:02 UT on 1 March 1985, i.e., about 2 minutes before the substorm onset (Speiser et al., 1996). For this distribution, the bulk flow was earthward and slightly duskward at $493 \mathrm{~km} / \mathrm{s}$, and the satellite was at the southern edge of the CS. It is this distribution that we concentrate on in this report. The new results we present here compare the model distribution function with the observations in cuts in the $v_{x}-v_{z}$ and $v_{y}-v_{z}$ planes, and show details of the particles' energization for the weak field and a neutral line. 


\section{The Neutral Line Model}

This AMPTE observed distribution is shown in Fig. 1 for three different cuts in velocity space $v_{x}-v_{z}$ (labelled $x-z$ ) and $v_{y}-v_{z}$, and $v_{x}-v_{y}$ with the contours indicated by the grey-shading and the value of the distribution function in SI units, i.e., $\mathrm{s}^{3} / \mathrm{m}^{6}$. Overlying the observed shaded contours are our modeled distribution contours to be discussed in the next section.

For the 02:40:02 distribution, in the earlier study we made model comparisons only for the cut in the $v_{x}-v_{y}$ plane. As before we follow 15,625 orbits and initialize with an assumed flowing source population.

The measured magnetic field is $21.4 \mathrm{nT}$ with elevation angle, $12^{\circ}$, and an azimuth angle, $-166^{\circ}$. Thus, the satellite is at the southern edge of the CS with $B_{x}=-20 \mathrm{nT}$, and $B_{y}=-5 \mathrm{nT}$. As in the previous event analyzed, we start with a final position on the CS edge, at various distances from a NL, and launch a large

\section{AMPTE $>$ IRM}

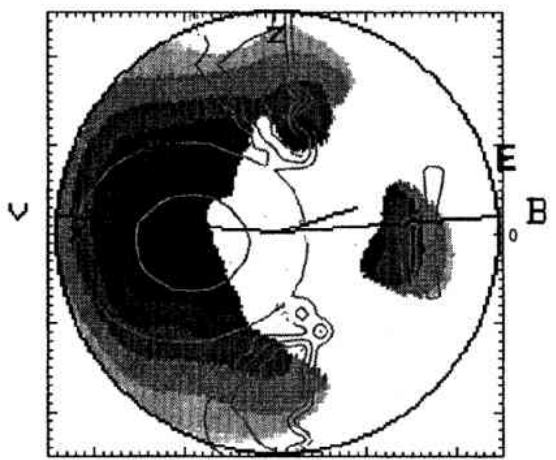

1 MAR 1985

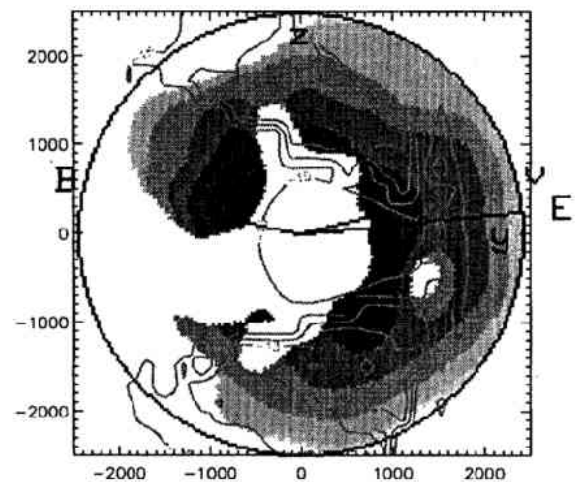

02: 40:02

$+1000$

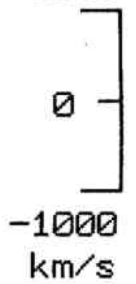

IONS

ด. $020-40.0 \mathrm{keV} / \mathrm{e}$

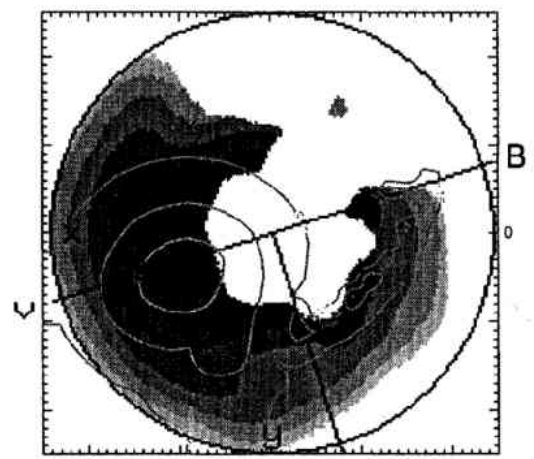

$10^{-15^{E}}$

$10^{-14}$

$10^{-13}$

Fig. 1. AMPTE/IRM observed distribution functions in 3 orthogonal velocity planes. $x-y$ corresponds to $v_{x}-v_{y}$, etc. Grey-scale bar at the bottom is for distribution function in $\mathrm{s}^{3} / \mathrm{m}^{6}$. Direction of observed $B, E$, and $V$ is indicated on the plots (see text). Velocity scale $(\mathrm{km} / \mathrm{s})$ is indicated on the $v_{x}-v_{z}$ (upper right) plot. Overlaid on each velocity plane slice in light grey are model distribution function contours $\left(10^{-15}, 10^{-14}, 10^{-13}\right)$ for the neutral line model. 
number of particles backwards in time, until they have ceased CS interaction, and tag them with an assumed initial distribution function. As with the previous case, we initially tried the model with $B_{x}=20$ $\mathrm{nT}, B_{y}=0, B_{z}=1 \mathrm{nT}, E_{y}=+0.25 \mathrm{mV} / \mathrm{m}$. Matching the model with Fig. 1 ( $v_{x}-v_{y}$ plot), we found that the $v_{x}$ offset of the centroid of the observation is adequately modeled again, using a strong earthward flow $(1,200 \mathrm{~km} / \mathrm{s})$, but somewhat smaller duskward flow $(400 \mathrm{~km} / \mathrm{s})$. In order to match the ridge structure seen in the data, a starting location at the CS edge and closer to the NL $\left(x_{0} \sim 0.4 L\right)$ was needed. The model contours $\left(-15,-14,-13\right.$ corresponding to $\left.10^{-15}, 10^{-14}, 10^{-13}\right)$ are overlaid on the $v_{x}-v_{y}$ plot of Fig. 1. Other model parameters are: CS half-thickness, $d=500 \mathrm{~km}$, separatrix dimension, $L=10,000 \mathrm{~km}$ (thus $x_{0} \sim 4,000$ $\mathrm{km}$, the distance from the observing point to the tailward neutral line), and for the source kappa distribution, $E_{\text {thermal }} \sim 2.4 \mathrm{keV}$ and $n_{\text {ion }}=3.67 \times 10^{5} / \mathrm{m}^{3}$. The kappa exponent used was 2 . For the distribution of Fig. 1, the measured bulk flow was $493 \mathrm{~km} / \mathrm{s}$ (elevation $=-4^{\circ}$, azimuth $=18^{\circ}$, temperature $=61.5$ $\times 10^{6} \mathrm{~K}(5.3 \mathrm{keV}), n_{\text {ion }}=0.442 \times 10^{6} / \mathrm{m}^{3}, n_{\text {high }}=0.18 \times 10^{6} / \mathrm{m}^{3}$ (density of ions with energies $\geq 8 \mathrm{keV}$ ) .

As noted previously for this comparison, the bulk of the distribution is fairly adequately modeled, and the "finger" in the $v_{x}<0, v_{y}>0$ quadrant almost magically appeared in the model when $B_{y}=-10 \mathrm{nT}$

\section{AHTTE $>$ IRH}

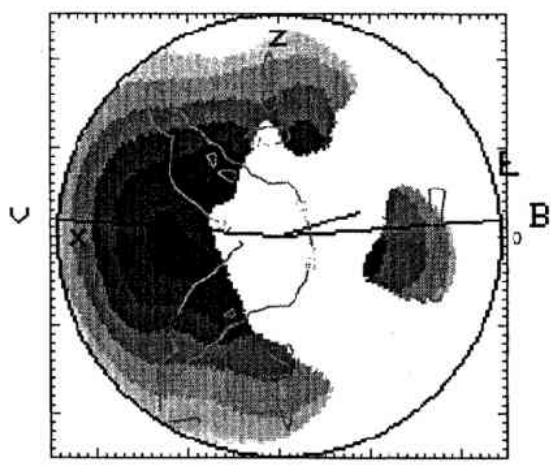

1 MAR 1985

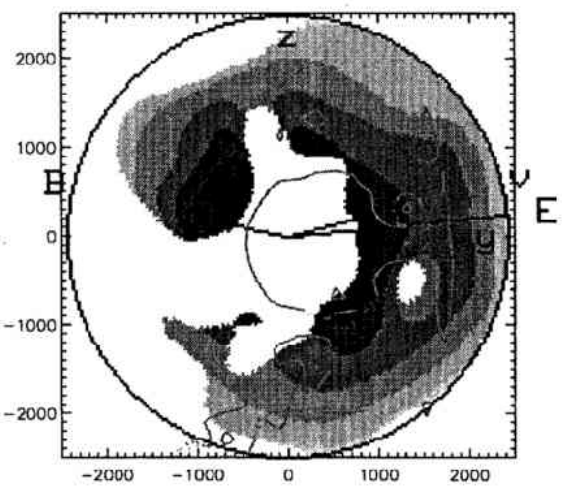

0อ: 40:02

$0.020-40.0 \mathrm{keV} / \mathrm{e}$

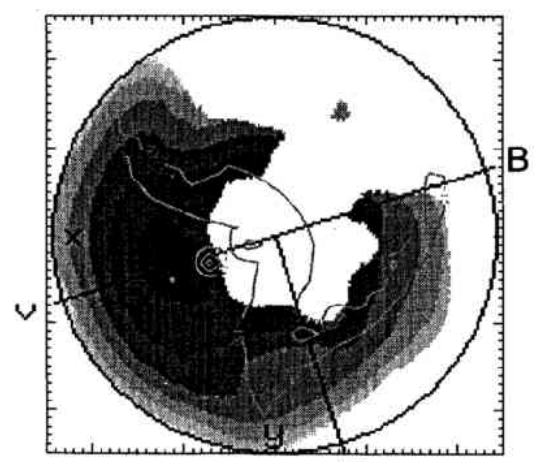

$10^{-15}$

$E$

Fig. 2. Same as Fig. 1, but with model contours from the weak field overlaid. 

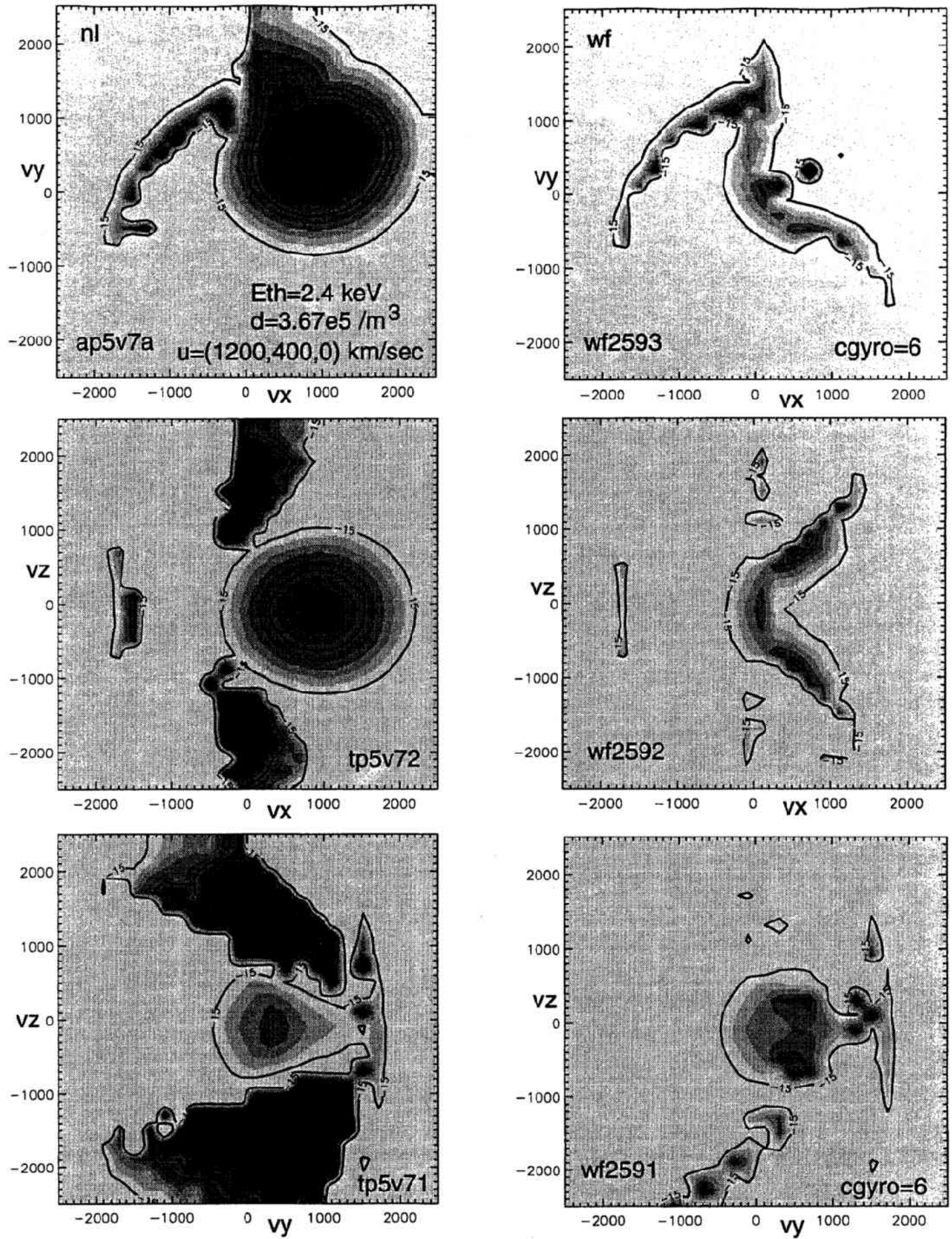

Fig. 3. Model distribution functions for the neutral line (left panels) and weak field (right panels) for an initial earthward and duskward flowing plasma. Three orthogonal slices top to bottom; $v_{x}-v_{y}, v_{x}-v_{z}$, and $v_{y}-v_{z}$. 


\section{initial energy (keV)}
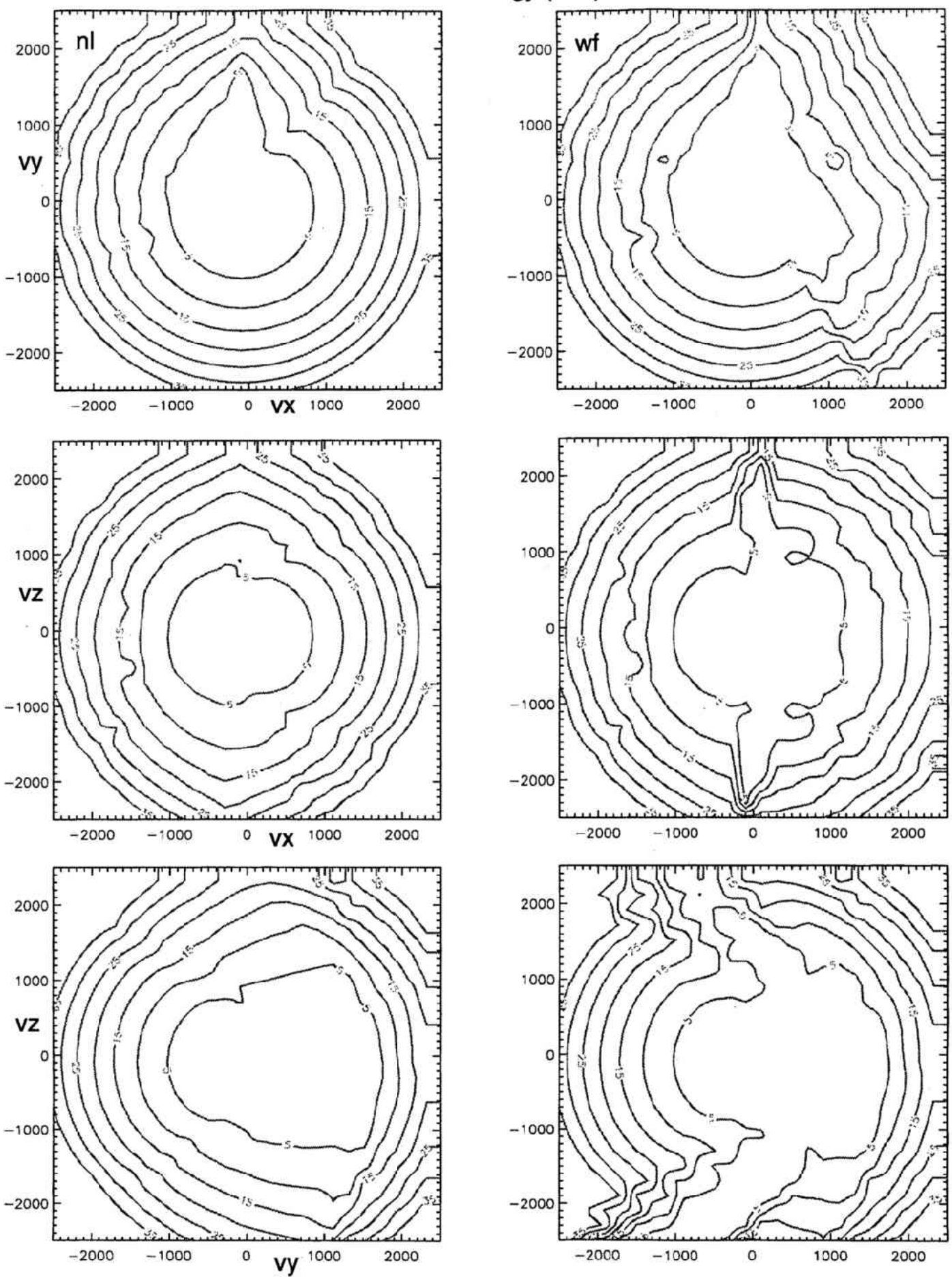

Fig. 4. Initial energy (keV) plots on the output velocity plane slices corresponding to Fig. 3 for the two models; neutral line left panels, and weak field right panels. 
was included (Speiser et al., 1996). The model input parameters we use throughout this study therefore add $B_{y}=-10 \mathrm{nT}$ to those mentioned in the previous paragraph. We did not try further for a "best fit". Note that while the model $B_{y}$ and the measured $B_{y}$ are both of the same sign, the magnitudes are different. The "finger" did not appear in the model for $B_{y}=-5 \mathrm{nT}$. This may indicate the non-local nature of the particle orbits. (The model contours, $-15,-14$ and -13 , are overlaid onto the AMPTE/IRM data in Fig. 1 as light grey contours.) The bulge in the -14 observation is approximately matched by the bulge in the -14 model contour in the $v_{x}>0, v_{y}>0$ quadrant. This is the ridge signature for ions that have been accelerated along the neutral line. The model models well most of the features in the $v_{x}-v_{y}$ plot, except for the second ridge seen in the observation in the $v_{x}>0, v_{y}<0$ quadrant. In the other slices in Fig. 1, again the model contours are overlaid over the observed distribution. In the $v_{x}-v_{z}$ slice (upper left, Fig. 1), the bulk flow is again well represented by the model, a feature near $v_{x}>0, v_{z} \approx 0$ is approximated, and lobes at $v_{x} \simeq 0, v_{y}>0$ and $v_{z}<0$ are fairly well modeled, although the model has more symmetry than the data. (More distribution function levels in the model can be seen in the 3 slices on the left-hand side of Fig. 3.) In the remaining panel (upper right, Fig. 1), the model contours are again overlaid on the AMPTE observations (see also Fig. 3). The centroid and the lobes of the observation are again approximated by the model, but this time not as well as in the other two slices. It is, however, interesting that the observed hole in the distribution at $v_{y} \sim 1,000 \mathrm{~km} / \mathrm{s}, v_{z} \sim-500 \mathrm{~km} / \mathrm{s}$ occurs where there is also a "hole" in the model distribution.

\section{Modeling a Weak Field Region}

Figure 2 is the same AMPTE/IRM distribution function, at 02:40:02 UT, as in Fig. 1, this time with model contours overlaid from a run with a weak field model. (Figure 3, right-hand side shows these model contours in more detail.) The weak field model has basically the same parameters as the neutral line model, $\boldsymbol{B}, \boldsymbol{E}, \mathrm{CS}$ thickness, source plasma, etc., except that the normal field component, $B_{z}$, does not reverse across the neutral line. That is ; $B_{z}=B_{0 z}|x| / d$ in the weak field region, rather than $B_{z}=B_{0 z} x / d$ as in the previous neutral line model.

In the $v_{x}-v_{y}$ panel (lower left, Fig. 2), the "finger region is still adequately modeled. This is because the particles in the "finger" region come from regions earthward of the neutral line, and the fields are the same in this region for the 2 models. The bulk flow of the observations is now poorly modeled and the other asymmetries (with one exception) are also poorly modeled. The exception is the second ridge seen in the observations in the $v_{x}-v_{y}$ slice in the $v_{x}>0, v_{y}<0$ quadrant. The weak field model now has a "tail" in this region that was absent in the neutral line model. This is one feature better modeled by the weak field model than by the neutral line model.

Figure 4 shows initial energy contours in $\mathrm{keV}$ for the 2 models (neutral line, left panels, weak field, right panels) for the three slices. Here for example, we can see outward bulges in the $v_{x}$ - $v_{y}$ slices for $v_{x} \approx 0, v_{y}>0$, i.e., indicative of acceleration along the neutral line for both models. Now, however, note the outer bulge on the contours for the weak field (WF) model in the $v_{x}>0, v_{y}<0$ quadrant. This corresponds to the second ridge and acceleration of ions along the weak field region. This is the reason for the "tail" appearing in the model distribution function as in Fig. 3, upper right panel. Other regions of acceleration (outward bulges) and deceleration (inward bulges) can be seen in the other slices of Fig. 4 .

\section{Discussion and Summary}

We have modeled 3D distribution functions observed by the AMPTE/IRM satellite near the center of the geomagnetic tail current sheet just after a moderate substorm onset, and at the current sheet edge at an earlier time. Ridge structures in the observed distribution function are consistent with the existence of a neutral line about one to one and a half $R_{e}$ tailward of the satellite (at $\left.(-12,-3,1) R_{e}\right)$, following substorm onset, and less than $1 R_{e}$ tailward prior to onset. Thus, according to our model, a near-Earth neutral line existed at least 1 to 2 minutes prior to the earliest indication of substorm activity at the ground. This mapping of the energetic ions thus allows one to use measured distributions to probe regions remote from 
the satellite. In order to map the centroid of the observation, we find that the initial distribution must have been streaming earthward at $\sim 1,200 \mathrm{~km} / \mathrm{s}$ and duskward at $\sim 400 \mathrm{~km} / \mathrm{s}$, thus it was probably pre-accelerated earthward in the more distant tail. Ground measurements indicate this would have been during a time of enhanced magnetospheric convection. The centroid position also seems to require a negative $B_{y}$ component in the range -5 to $-10 \mathrm{nT}$. This agrees with the locally measured $B_{y}$. Such a component also affects the dynamical nature of the particle orbits and produces a "finger" in the distribution that is quite similar to the observations. This feature is gyro-phase dependent according to the model (Speiser et al., 1996). Thus, certain features of the observed distribution function may be due to gyro-phase selectivity! While there may be other mechanisms capable of reproducing some of the observations, we know of no other mechanism which is able to reproduce several features simultaneously. A weak field model without a reversing $B_{z}$ neutral line reproduces the "finger" and ridge fairly well, but the centroid only poorly. However, the weak field model does produce a second ridge in a different portion of velocity space which approximately models a second ridge seen in the observations, and which is not modeled by the neutral line model. Ions are accelerated along this second, or weak field ridge, in the weak field model, but not in the neutral line model. Thus, while the observed AMPTE/IRM distribution indicates the existence of a neutral line near the Earth 2 minutes prior to substorm onset, it is possible that a mixed weak field ridge signature is caused by the field changing from a neutral line to a weak field in time, while the ions moving toward their encounter with the AMPTE/IRM instrument are moving through the fields. We have previously shown that ions making up one of these modeled distributions take up to 80 seconds from source to observation point (Martin and Speiser, 1994). Therefore, a field fluctuating between neutral line and weak field on about a 1 minute time scale could give rise to these mixed neutral line and weak field signatures.

The field we use in our simulations is stationary in time. If the field does change from a neutral line to a weak field, we can estimate the induced field from Faraday's Law. Then,

$$
\Delta E_{y}=\int \frac{d B_{z}}{d t} d x \approx \int \frac{\Delta B_{0 z}}{\Delta t} d x \approx \int \frac{B_{w f}-B_{n l}}{\Delta t} d x,
$$

where $B_{w f}-B_{n l}$ is the difference between weak field and neutral line $z$-components. Thus,

$$
\Delta E_{y} \approx \frac{\Delta B_{z}}{L \Delta t} \int_{0}^{L}(|x|-x) d x
$$

But the integral is zero for $x>0$, and for $x<0$, goes to $L^{2} / 2$. So

$$
E_{y} \approx \frac{B_{0 z} L}{\Delta t} \sim 2 \times 10^{-4} \mathrm{~V} / \mathrm{m} \quad(\text { i.e., } \approx 0.2 \mathrm{mV} / \mathrm{m})
$$

Thus, the induced field is zero for $x>0$, i.e. for particles falling on the CS earthward of the neutral line. All of the particles which make up the "finger" region for example come from $x>0$ (Speiser et al., 1996), and would be unaffected. However for $x<0$, this estimate implies the induced electric field could be of the same order as our applied $E_{y}(.25 \mathrm{mV} / \mathrm{m})$. In our previous study (Martin et al., 1994) we found the dynamics not to be terribly sensitive to a different electric field model. Thus, extension of these models to include time dependency is probably worth investigating, but we believe it is unlikely to affect our model distributions in a significant way.

We thank Eigel Friis-Christensen, Gordon Rostoker, and Howard Singer for magnetometer data, Goetz Paschmann and Norbert Sckopke for the AMPTE data, and Margaret Kivelson, Bob McPherron, Gordon Rostoker, and Howard Singer for helpful discussions. 
This research has been supported by the National Science Foundation (ATM-9002447, ATM-9301801, ATM9496294), and by the National Aeronautics and Space Administration (NAGW-1054, NAGW-1176, NAGW-2968, NAG5-1506).

\section{REFERENCES}

Angelopoulos, V., W. Baumjohann, C. F. Kennel, F. V. Coroniti, M. G. Kivelson, R. Pellat, R. J. Walker, H. Lühr, and G. Paschmann, Bursty bulk flows in the inner central plasma sheet, J. Geophys. Res., 97, 4027-4039, 1992.

Baumjohann, W., G. Paschmann, and H. Lühr, Characteristics of high-speed ion flows in the plasma sheet, J. Geophys. Res., 95, 3801-3809, 1990.

Dusenbery, P., G. Burkhart, and T. Speiser, The effect of a nonzero $B_{y}$ field on particle motion in the tail, Geophys. Res. Lett., 1996 (to be submitted).

Huang, C. Y., L. A. Frank, and T. E. Eastman, Plasma flows near the neutral sheet of the magnetotail, in Magnetotail Physics, edited by A. T. Y. Lui, pp. 128, John Hopkins University Press, Baltimore, Maryland, 1987.

Martin, R., Jr. and T. Speiser, A predicted energetic ion signature of a neutral line in the geomagnetic tail, J. Geophys. Res., 93, $11,521-11,526,1988$.

Martin, R., Jr. and T. Speiser, Model particulaire d'une bouffée d'écoulement, in Recueil D'Actes du Seminaire du GDR Plasmae, France, 1994.

Martin, R., Jr., T. Speiser, and K. Klamczynski, The effect of $B_{y}$ on neutral line ridges and dynamical source ordering, J. Geophys. Res., 99, 23,623-23,638, 1994.

Nakamura, M., G. Paschmann, W. Baumjohann, and N. Sckopke, Ion distributions and flows near the neutral sheet, J. Geophys. Res., 96, 5631-5649, 1991.

Speiser, T. and R. Martin, Jr., Energetic ions as remote probes of $x$-type neutral line ridges in the geomagnetic tail, J. Geophys. Res., 97, 10,775-10,785, 1992.

Speiser, T. and R. Martin, Jr., Neutral line energetic ion signatures in the geomagnetic tail: Comparisons with AMPTE observations, in Proceedings of the A GU Chapman Conference on Micro-Meso Scale Phenomena in Space Plasmas, Geophys. Monog. 86, pp. 243-253, edited by N. Ashour-Abdalla, T. Chang, and P. Dusenbery, Amer. Geophys. Un., Washington, D.C., 1995.

Speiser, T., R. Martin, Jr., and N. Sckopke, Bursty bulk flows, the geomagnetic tail current sheet, and substorm timing, Adv. Space Res., 18, No. 8, 73-78, 1996. 